\title{
Severe Aortic stenosis: Is urgent transcatheter aortic valve replacement better than Balloon aortic valvuloplasty better than?
}

\author{
Wael Awad ${ }^{1}$, Mohammed Idhrees ${ }^{2}$, and Mohamad Bashir ${ }^{3}$ \\ ${ }^{1}$ St Bartholomew's Hospital \\ ${ }^{2}$ SRM Institutes for Medical Science Vadapalani \\ ${ }^{3}$ Royal Blackburn Teaching Hospital
}

October 26, 2020

\section{Severe Aortic stenosis: Is urgent transcatheter aortic valve replacement better than Balloon aortic valvuloplasty better than?}

Wael Awad FRCS(C/Th) ${ }^{1}$, Mohammed Idhrees MS, MCh, FAIS ${ }^{2}$, Mohamad Bashir MD, PhD, MRCS ${ }^{3}$

1. Department of Cardiothoracic Surgery, Barts Heart Centre, St. Bartholomew's Hospital,

West Smithfield, London, UK

2. Institute of Cardiac and Aortic disorders, SRM Institues for Medical Science (SIMS Hospital), India

3. Department of Vascular Surgery, Royal Blackburn Teaching Hospital, Haslingden Road,

Blackburn, UK

\section{Corresponding Author:}

Wael I Awad MD FRCS(C/Th)

Department of Cardiothoracic Surgery

Barts Heart Centre, St Bartholomew's Hospital

West Smithfield

London EC1A 7BE

UK

Tel: +442037658744

E-mail: wael.awad@nhs.net

Key words: Transcatheter aortic valve replacement, severe aortic stenosis, Balloon aortic valvuloplasty

Word Count: 1931 words

Article Type: Invited Commentary

Elective TAVR versus Urgent TAVR

Bianco et al. [1] report a single centre retrospective analysis of 1193 patients that underwent transcatheter aortic valve replacement (TAVR) over an 8-year period from 2011-2018; of which 247 (20.7\%) were urgent and 946 were elective procedures. The authors compared the urgent and elective procedure and studied 
in-hospital, short and mid-term survival and hospital readmissions. They reported that the 30 day mortality ( $6.5 \%$ vs $2.3 \%)$, acute kidney injury ( $2.8 \%$ vs $0.6 \%)$ and length of stay (12 vs 3 days) were all significantly higher in the urgent group vs those having elective TAVR procedures, respectively.

Freedom from readmission for heart failure at 1-year was lower for the urgent group (73.6\% vs $83.4 \%)$, and the 1-year $(79.0 \%$ vs $87.1 \%)$ and 5 -year (39.6\% vs $43.5 \%)$ survival was lower in this group vs the elective group, although this difference was eliminated after risk adjustment. The authors conclude although urgent TAVR is associated with increased periprocedural risk due to more co-morbid disease, outcomes and long-term survival support the consideration of urgent TAVR as a viable alternative for this patient population.

This is an important topic for cardiologists and cardiac surgeons because of the relative frequency of patients with severe aortic stenosis (AS) admitted to hospital with related symptoms and heart failure. Although most patients with heart failure can be medically managed with subsequent discharge and elective intervention, this may put them at a higher risk for recurrent heart failure and readmission. Moreover, acute heart failure and cardiogenic shock in severe AS are associated with poor prognoses, as well as an extremely high operative risk for surgical aortic valve replacement (SAVR) [2-4].

Institutional practices, local and logistic factors can affect patient selection and management approaches to severe aortic stenosis. Although TAVR is generally performed on an appropriate basis, there is still a need to determine how to best manage the list for TAVR as well as to develop benchmarks for the maximum acceptable waiting time for patients with severe AS pending intervention. In addition, the potential benefit of TAVR needs to be weighed against the periprocedural risks and the likelihood of futility.

\section{Balloon aortic valvuloplasty - still an option?}

Balloon aortic valvuloplasty (BAV) remains an option for temporary palliation and symptomatic relief in such patients. However, long-term survival after BAV alone remains poor, with a high occurrence of valvular restenosis[5]. BAV can also play an important role as a bridge to either surgical or TAVR in patients with AS requiring temporary haemodynamic stabilization. However, there may be significant delays between BAV and AVR or TAVR[6]. In a multicenter registry of 811 patients with severe AS who underwent BAV, at a median follow-up of 318 days, only $30.9 \%$ of patients undergoing BAV as a bridge to TAVR and $15.8 \%$ of patients undergoing BAV as a bridge to SAVR actually underwent AVR[7]. Additionally in this study, $56.5 \%$ of patients who underwent urgent/emergent TAVR had a prior history of BAV, suggesting that BAV may not be effective in preventing subsequent acute decompensation and need for urgent/emergent TAVR.

Ali et al. [8] compared strategies in the treatment of decompensated severe aortic stenosis. The authors hypothesised that undertaking urgent or emergency TAVR directly in such patients is safer and more effective than urgent or emergency balloon aortic valvuloplasty (BAV) followed by elective TAVR or surgical aortic valve replacement (SAVR). Between September 2014 and February 2018, 52 patients underwent urgent or emergency BAV and 87 underwent TAVR. Significant differences were noted between the two groups in 30-day all-cause mortality (88.5\% BAV patients alive at 30 days, $97.7 \%$ TAVR patients) and 1-year all-cause mortality (44.2\% BAV patients alive at 1 year, $88.5 \%$ TAVR patients). Patients in the BAV group who successfully underwent subsequent TAVR or SAVR all survived for 365 days, but there was no significant 1year mortality difference compared with those who underwent urgent or emergency TAVR (100 vs. $88.5 \%$; P $>0.155)$. These results suggest treatment of decompensated severe aortic stenosis with urgent or emergency TAVR may be associated with improved survival outcomes when compared with a strategy of performing BAV as a bridge to subsequent TAVR or SAVR.

Kolte et al.[9] examined outcomes and identified independent predictors of mortality among patients undergoing urgent/emergent TAVR. The Society of Thoracic Surgeons and the American College of Cardiology Transcatheter Valve Therapy (STS/ACC TVT) Registry linked with Centers for Medicare \& Medicaid Services claims was used to identify patients who underwent urgent/emergent versus elective TAVR between November 2011 and June 2016. Of 40,042 patients who underwent TAVR, 3,952 (9.9\%) were urgent/emergent. Device success rate was statistically lower, after urgent/emergent versus elective TAVR (92.6\% vs. 93.7\%). Rates of major and/or life-threatening bleeding, major vascular complications, myocardial infarction, stroke, 
new permanent pacemaker placement, conversion to SAVR, and paravalvular regurgitation were similar between the 2 groups. Compared with elective TAVR, patients undergoing urgent/emergent TAVR had higher rates of acute kidney injury (AKI) and/or new dialysis ( $8.2 \%$ vs. $4.2 \%), 30$-day mortality $(8.7 \%$ vs. $4.3 \%$, , and 1-year mortality ( $29.1 \%$ vs. $17.5 \%)$. The authors conclude that urgent/emergent TAVR is feasible with acceptable outcomes and may be a reasonable option in a selected group of patients with severe AS.

AKI and new dialysis are more common following urgent/emergent versus elective TAVR. The causes of AKI after TAVR are many including hypotension/hypoperfusion during rapid pacing, contrast-induced AKI (CIAKI), bleeding and blood transfusions. Pre-procedure renal dysfunction, diabetes, impaired left ventricular function, are also more common in patients undergoing urgent/emergent TAVR and are associated with an increased risk of new dialysis after TAVR[11] Furthermore, patients requiring urgent/emergent TAVR may undergo pre-operative CT scans and cardiac catheterization within a short period of time, increasing the risk of CIAKI. However, after adjusting for baseline patient and procedural characteristics, Ferro et al. [11]found no significant difference in the adjusted odds of AKI/new dialysis between urgent/emergent vs elective TAVR, suggesting that the observed differences in the rates are related to differences in baseline clinical risk profile rather than the procedure itself. Use of 3-dimensional transoesophageal echocardiography and non-contrast imaging for pre-procedural AV annulus assessment may help decrease the risk of CIAKI/new dialysis in patients at increased risk of this complication, including those undergoing urgent/emergent TAVR[12-14].

In patients undergoing urgent/emergent TAVR, oxygen-dependent lung disease, immunocompromised status, pre-existing atrial fibrillation/flutter, higher baseline creatinine, concomitant mitral stenosis, non-femoral access, are associated with an increased risk of 1-year mortality. Several of these variables have also been shown to predict poor outcome following TAVR[15-16]. Thus, TAVR, especially as an urgent/emergent procedure, might be considered medically futile in patients with 1 or more of these comorbidities and such patients should be considered for emergency BAV as a palliative therapy or bridge to decision.

\section{Conclusions}

In patients with severe aortic valve stenosis presenting acutely, the haemodynamic instability and resistance to optimized drug therapy may require an urgent intervention. BAV remains a viable treatment option. Outcomes in patients bridged to AVR/TAVI are better than in patients treated with BAV alone. Owing to the high mortality of patients in this cohort without destination therapy, delays to progression to TAVR or AVR should be avoided. Urgent or emergency TAVR may be associated with improved survival outcomes when compared with BAV as a bridge to subsequent TAVR or SAVR. Urgent TAVR may be associated with increased peri-procedural risk but outcomes are acceptable and this approach is a viable alternative for this patient population.

\section{References:}

1. Valentino Bianco et al. Urgent Transcatheter Aortic Valve Replacement may be performed with acceptable long-term outcomes.

2. Carabello BA, Green LH, Grossman W, Cohn LH, Koster JK, Collins JJ Jr. Hemodynamic determinants of prognosis of aortic valve replacement in critical aortic stenosis and advanced congestive heart failure. Circulation. 1980 Jul;62(1):42-8. doi: 10.1161/01.cir.62.1.42.

3. Piérard S, de Meester C, Seldrum S, Pasquet A, Gerber B, Vancraeynest D, Robert A, El Khoury G, Noirhomme P, Vanoverschelde JL. Impact of preoperative symptoms on postoperative survival in severe aortic stenosis: implications for the timing of surgery. Ann Thorac Surg. 2014 Mar;97(3):803-9. doi: 10.1016/j.athoracsur.2013.08.059.

4. Nagao K, Taniguchi T, Morimoto T, Shiomi H, et al. CURRENT AS Registry Investigators. Acute Heart Failure in Patients With Severe Aortic Stenosis - Insights From the CURRENT AS Registry. Circ J. 2018 Feb 23;82(3):874-885. doi: 10.1253/circj.CJ-17-0610.

5. Ben-Dor I, Pichard AD, Satler LF, Goldstein SA, et al. Complications and outcome of balloon aortic 
valvuloplasty in high-risk or inoperable patients. JACC Cardiovasc Interv. 2010 Nov;3(11):1150-6. doi: 10.1016/j.jcin.2010.08.014.

6. Saia F, Marrozzini C, Ciuca C, Guastaroba P, Taglieri N, et al. Emerging indications, in-hospital and long-term outcome of balloon aortic valvuloplasty in the transcatheter aortic valve implantation era. EuroIntervention. 2013 Apr 22;8(12):1388-97. doi: 10.4244/EIJV8I12A212.

7. Moretti C, Chandran S, Vervueren PL, D'Ascenzo F, Barbanti M, et al. Outcomes of Patients Undergoing Balloon Aortic Valvuloplasty in the TAVI Era: A Multicenter Registry. J Invasive Cardiol. 2015 Dec;27(12):547-53.

8. Ali N, Patel P, Wahab A, Das A, Blackman DJ, et al. A cohort study examining urgent and emergency treatment for decompensated severe aortic stenosis. J Cardiovasc Med (Hagerstown). 2020 Sep 15. doi: 10.2459/JCM.0000000000001112.

9. Kolte D, Khera S, Vemulapalli S, Dai D, Heo S, et al. Outcomes Following Urgent/Emergent Transcatheter Aortic Valve Replacement: Insights From the STS/ACC TVT Registry. JACC Cardiovasc Interv. 2018 Jun 25;11(12):1175-1185. doi: 10.1016/j.jcin.2018.03.002.

10. Nishimura RA, Otto CM, Bonow RO, Carabello BA, Erwin JP 3rd, et al. 2017 AHA/ACC Focused Update of the 2014 AHA/ACC Guideline for the Management of Patients With Valvular Heart Disease: A Report of the American College of Cardiology/American Heart Association Task Force on Clinical Practice Guidelines. Circulation. 2017 Jun 20;135(25):e1159-e1195. doi: 10.1161/CIR.0000000000000503.

11. Ferro CJ, Law JP, Doshi SN, de Belder M, Moat N, et al. Dialysis Following Transcatheter Aortic Valve Replacement: Risk Factors and Outcomes: An Analysis From the UK TAVI (Transcatheter Aortic Valve Implantation) Registry. JACC Cardiovasc Interv. 2017 Oct 23;10(20):2040-2047. doi: $10.1016 /$ j.jcin.2017.05.020.

12. Prihadi EA, van Rosendael PJ, Vollema EM, Bax JJ, Delgado V, et al. Feasibility, Accuracy, and Reproducibility of Aortic Annular and Root Sizing for Transcatheter Aortic Valve Replacement Using Novel Automated Three-Dimensional Echocardiographic Software: Comparison with Multi-Detector Row Computed Tomography. J Am Soc Echocardiogr. 2018 Apr;31(4):505-514.e3. doi: 10.1016/j.echo.2017.10.003.

13. Ruile P, Blanke P, Krauss T, Dorfs S, Jung B, et al. Pre-procedural assessment of aortic annulus dimensions for transcatheter aortic valve replacement: comparison of a non-contrast 3D MRA protocol with contrast-enhanced cardiac dual-source CT angiography. Eur Heart J Cardiovasc Imaging. 2016 Apr;17(4):458-66. doi: 10.1093/ehjci/jev188.

14. Wang J, Jagasia DH, Kondapally YR, Herrmann HC, Han Y. Comparison of Non-Contrast Cardiovascular Magnetic Resonance Imaging to Computed Tomography Angiography for Aortic Annular Sizing Before Transcatheter Aortic Valve Replacement. J Invasive Cardiol. 2017 Jul;29(7):239-245.

15. Arnold SV, Reynolds MR, Lei Y, Magnuson EA, Kirtane AJ, et al. Predictors of poor outcomes after transcatheter aortic valve replacement: results from the PARTNER (Placement of Aortic Transcatheter Valve) trial. Circulation. 2014 Jun 24;129(25):2682-90. doi: 10.1161/CIRCULATIONAHA.113.007477.

16. Arnold SV, Afilalo J, Spertus JA, Tang Y, Baron SJ, et al. Prediction of Poor Outcome After Transcatheter Aortic Valve Replacement. J Am Coll Cardiol. 2016 Oct 25;68(17):1868-1877. doi: 10.1016/j.jacc.2016.07.762. 